\section{OWE-20 SURVIVAL \& FUNGAL CATHETER RELATED BLOOD STREAM INFECTIONS IN PATIENTS ON HPN}

${ }^{1,2} \mathrm{SM}$ Oke*, ${ }^{2} \mathrm{~N}$ Miah, ${ }^{1} \mathrm{~N}$ Fourali, ${ }^{1} \mathrm{P}$ Khanna, ${ }^{1} \mathrm{SC}$ Donnelly, ${ }^{1} \mathrm{M}$ Small, ${ }^{1} \mathrm{SM}$ Gabe. ${ }^{1} \mathrm{St}$ Mark's Hospital, Harrow, UK; ${ }^{2}$ Imperial College London, London, UK

10.1136/gutjnl-2019-BSGAbstracts.330

Introduction Candidiasis is associated with significant morbidity \& mortality. Central venous catheters (CVC) are commonly used for home parenteral nutrition (HPN) in patients with intestinal failure (IF) \& are commonly linked to development of candidaemia. We aim to describe the long-term survival in patients with candidal catheter related blood stream infections (CRBSI) in the IF population \& assess factors that may be associated with poorer outcome.

Methods Clinical records of patients receiving HPN at our unit were reviewed between $1 / 1 / 2007$ to $1 / 5 / 2018$. Candidal CRBSI was defined as either: documented or reported positive cultures from the CVC line with negative peripheral cultures, peripheral cultures with time to positivity faster than CVC cultures, paired positive cultures from CVC \& peripheral blood with symptoms of sepsis, or positive CVC tip culture following CVC removal.

Demographic data, underlying aetiology, small intestinal length, colonic continuity, long-term survival \& cause of death were recorded. Data were also collated on number of candidal CRBSI, candidal species, bacterial co-infection, antifungal resistance together with opiate $\&$ immunosuppression use at the time of infection. Differences between groups were analysed using Chi-Squared tests, Kaplan-Meier \& Cox's regression model were used to assess factors affecting survival.

Results 66 patients were identified of whom 22 (33.3\%) were male. The mean age was 49.4 (SD 16.5). 13 (19.7\%) patients had multiple candidal CRBSI. Candida galbrata \& albicans accounted for $32(48.5 \%)$ of the index infections. $8(12 \%)$ patients had candidal species that were resistant to $\geq 1$ antifungal agent. $48(72.7 \%)$ patients were receiving an opiate at the time of the fungal CRBSI, \& 8 (12.1\%) were receiving some form of immunosuppression.

$25(38 \%)$ patients died during the study period. The 1, 5 $\& 10$ year survival rates were 97\%, 67\% \& 53\% respectively. From the time of first fungal CRBSI, the $1,5 \& 10$ year survival rates were $85 \%, 55 \% \& 27 \%$. No difference was seen in survival between patients who had single \& multiple candidal infections (log-rank $\mathrm{p}=0.669)$. On multivariate analysis increasing age (HR $1.08 \mathrm{p}=0.02)$, increasing time of HPN prior to index infection (HR $0.45 \mathrm{p}<0.005$ ), length of residual small intestinal length (HR $0.76 \mathrm{p}=0.06)$ underlying aetiology (HR 6.40 p=0.017) \& opiate use (HR $39.5 \mathrm{p}=0.003$ ) were all associated with long-term survival.

Conclusion Our data have shown long-term survival after index fungal CRBSI in the IF population are poor. A better prognosis is associated with a small intestinal length of $\geq$ $150 \mathrm{~cm} \&$ increasing length of time on HPN prior to first infection. A worse prognosis is associated with underlying aetiology including mesenteric infarction \& scleroderma \& the use of opiates.

\section{OWE-36 PREVALENCE AND FACTORS ASSOCIATED WITH IMPAIRED FOOD-RELATED QUALITY OF LIFE IN 1223 PEOPLE WITH IBD}

${ }^{1}$ Wladyslawa Czuber-Dochan*, 'Trevor Murrells, "Myfanwy Morgan, 'Miranda Lomer, ${ }^{2}$ James $\mathrm{O}$ Lindsay, ${ }^{1}$ Kevin Whelan. ${ }^{1}$ King's College London, London, UK; ${ }^{2}$ Barts Health NHS Trust, London, UK

\subsection{6/gutjnl-2019-BSGAbstracts.331}

Introduction Inflammatory bowel disease (IBD) patients often report that dietary intake and the enjoyment of food is affected by their condition. However, the prevalence of impaired food-related quality of life (FR-QoL) and associated factors have not been previously explored. This study aimed to determine the levels of FR-QoL and factors associated with it in a large, nationally representative sample of people with IBD.

Methods A convenience sample of 1576 IBD outpatients $\geq 16$ years old were recruited from seven UK centres. Patients consuming the majority of their intake as food completed previously validated questionnaires to capture demographic data, FRQoL-29, quality of life (IBDQ UK), IBD-distress (IBD-DS), IBD-fatigue (IBD-F), and anxiety and depression (HADS). A health professional recorded disease activity (HBI, SCCAI), disease classification (Montreal), blood results, body mass index and malnutrition risk (MUST). FR-QoL was regressed onto the explanatory variables (univariable/multivariable) using the Stata MI (20 imputed datasets) procedure.

Results Data from 1223 patients were available $(78 \%$ response, $65 \% \mathrm{CD}$ and $51 \%$ female). FR-QoL mean score was 80.1 [SD 26.9] (minimum 29, maximum 145, higher score $=$ better FR-QoL), considerably lower in comparison to previously measured healthy volunteers [123.0, SD 16.5]. The four items rated as the most severe (Strongly agree/Agree) were 'avoiding food and drink I know does not agree with my IBD' (71\%), 'being more aware of what I am eating due to my IBD' (70\%), 'certain foods have triggered symptoms of my IBD' (69\%) and 'enjoyment of a particular food or drink has been affected by the knowledge that it might trigger my IBD symptoms' (67\%). Twenty-six factors (demographic, clinical, drug-related, psycho-social) were significantly associated with impaired FR-QOL in univariate analysis. However, in the multivariable regression only lower educational level $(\mathrm{p}<.001)$, greater number of IBD flares in last two years $(p<.001)$, more severe symptoms during last flare $(\mathrm{p}=.034)$, not taking immune suppressants $(p=.026)$, greater distress $(p<.001)$, greater fatigue impact on daily living $(p=.025)$ and worse IBD QoL $(\mathrm{p}<.001)$ remained significantly associated with impaired FR-QoL.

Conclusions In this first large study reporting FR-QoL in IBD, many factors were identified as having a significant negative effect on patients with IBD. Understanding the relationship between IBD and FR-QoL may improve communication between health professionals and patients regarding its impact. 